\title{
Surface diffusion of long chainlike molecules: The role of memory effects and stiffness on effective diffusion barriers
}

\author{
Hjelt, T.; Vattulainen, Ilpo Tapio
}

Published in:

Journal of Chemical Physics

Link to article, DOI:

$10.1063 / 1.481029$

Publication date:

2000

Document Version

Publisher's PDF, also known as Version of record

Link back to DTU Orbit

Citation (APA):

Hjelt, T., \& Vattulainen, I. T. (2000). Surface diffusion of long chainlike molecules: The role of memory effects and stiffness on effective diffusion barriers. Journal of Chemical Physics, 112(10), 4731-4738.

https://doi.org/10.1063/1.481029

\section{General rights}

Copyright and moral rights for the publications made accessible in the public portal are retained by the authors and/or other copyright owners and it is a condition of accessing publications that users recognise and abide by the legal requirements associated with these rights.

- Users may download and print one copy of any publication from the public portal for the purpose of private study or research.

- You may not further distribute the material or use it for any profit-making activity or commercial gain

- You may freely distribute the URL identifying the publication in the public portal

If you believe that this document breaches copyright please contact us providing details, and we will remove access to the work immediately and investigate your claim. 


\title{
Surface diffusion of long chainlike molecules: The role of memory effects and stiffness on effective diffusion barriers
}

\author{
T. Hjelt \\ Helsinki Institute of Physics, P.O. Box 9 (Siltavuorenpenger 20 C), FIN-00014 University of Helsinki, \\ Finland \\ I. Vattulainen \\ Department of Chemistry, Technical University of Denmark, Building 207, DK-2800 Lyngby, Denmark
}

(Received 21 June 1999; accepted 13 December 1999)

\begin{abstract}
We study the coverage dependence of surface diffusion for chainlike molecules by the fluctuating-bond model with a Monte Carlo dynamics. The model includes short-ranged excluded volume interactions between different chains as well as an intrachain bond angle potential to describe the chain stiffness. Our primary aim is to consider the role played by chain stiffness and the resulting memory effects in tracer diffusion, and in particular their role in the effective tracer diffusion barrier $E_{A}^{T}$ extracted from the well-known Arrhenius form. We show that the memory effects in tracer diffusion become more pronounced at an increasing coverage as a result of packing requirements. Increasing the chain flexibility furthermore has the same overall effect as increasing the chain length, namely, they both increase $E_{A}^{T}$. We then analyze the influence of memory effects on $E_{A}^{T}$ and find that, for a single diffusing chain, about $20 \%$ of $E_{A}^{T}$ arises from temperature variations in the memory effects, while only the remaining part comes from thermally activated chain segment movements. At a finite coverage, the memory contribution in $E_{A}^{T}$ is even larger and is typically about $20 \%-40 \%$. Further studies with chains of different lengths lead to a conclusion that, for a single diffusing chain, the memory contribution in $E_{A}^{T}$ decreases along with an increasing chain length and is almost negligible in the case of very long chains. Finally, we close this work by discussing our results in light of recent experimental work as regards surface diffusion of long DNA molecules on a biological interface. (C) 2000 American Institute of Physics. [S0021-9606(00)70110-3]
\end{abstract}

\section{INTRODUCTION}

Surface diffusion plays a fundamental role in various physically and technologically important processes such as chemical reactions in the purification of exhaust gases and surface growth for the semiconductor industry. ${ }^{1,2}$ Increasing effort has therefore been devoted to understand the basic principles that govern diffusion. ${ }^{1-3}$ In the case of diffusion of single atoms at very low coverages, significant steps forward have indeed been made. One example of this progress is the experimental $^{4,5}$ and simulation work ${ }^{5,6}$ that has established a considerable understanding of various mechanisms by which single atoms diffuse on solid surfaces. However, in spite of the progress in the single-atom case, much less is known about surface diffusion of more complex particles such as chainlike molecules. This is mainly due to the complexity of these molecules, which gives rise to complex diffusion mechanisms that involve coupled translational and rotational degrees of freedom as well as a strong dependence on the molecular size, molecular shape, the coverage, and the mismatch between the molecule and the surface. ${ }^{7-18}$ Most work done so $\operatorname{far}^{7-16}$ has focused on the diffusion properties of short, single molecules. Nevertheless, it should be stressed that in various technologically important processes that involve thin films, such as lubrication and coating, one is interested in many-chain diffusion of long molecules, which is obviously a much more complicated issue.

Of special interest has also been the temperature depen- dence of the tracer diffusion coefficient, which is characterized by the diffusion barrier $E_{A}^{T}$ extracted from an Arrhenius analysis. Molecular dynamics simulations ${ }^{7-9,11,12,16,17}$ and experiments $^{19-22}$ have revealed that diffusion of short chainlike molecules can often be described by the Arrhenius description. Unfortunately, however, the actual microscopic origins of the effective activation barrier are poorly understood. Supposedly the diffusion barrier is somehow related to interactions between the chain and the substrate as well as interactions between the chains themselves. However, since consecutive displacements of chain segments are correlated and these dynamical correlations (memory effects) are temperature dependent, ${ }^{23}$ the memory effects are also contributing to the diffusion barrier. In real systems all these different contributions are mixed altogether, and therefore a microscopic interpretation of $E_{A}^{T}$ is very difficult to make, if possible at all. In this respect, studies of model systems can provide a great deal of insight into the understanding of the diffusion barriers.

The purpose of this work is to systematically consider the surface diffusion of long chainlike molecules over a wide coverage range. Our primary aim is to assess the importance of memory effects in the diffusion barriers of chains, whose stiffness is varied between stiff and flexible regimes. Obviously, this would be a very tedious task if done by molecular dynamics. The fluctuation-bond model $^{24,25}$ employed here, however, suits for this purpose very well. In this model, a 
number of structural units (corresponding to the persistence length) in a real linear molecule are replaced with a single model segment, which allows an efficient mapping ${ }^{25,26}$ from a real chain to a coarse-grained model system. We employ this model to a study of tracer diffusion via Monte Carlo simulations, when the chain stiffness is controlled by an intrachain bond-angle potential. Using a formal decomposition of the tracer diffusion coefficient $D_{T},{ }^{23,27}$ we then extract the contribution of memory effects from the data as contained in the corresponding correlation factor. For stiff as well as flexible chains, we find the memory effects to manifest themselves as back-correlations; i.e., after an initial movement, a chain segment tends to move backwards towards its previous site. This effect increases in the presence of other chains, which leads to an overall conclusion that the memory effects become more important with an increasing coverage. As far as the temperature dependence of memory effects is concerned, we find its role to be most pronounced in flexible chains. In this case, up to about $40 \%$ of the effective diffusion barrier $E_{A}^{T}$ of tracer diffusion arises from temperature variations in the memory effects, while only the remaining part comes from thermally activated chain segment movements. In stiff chains, the memory contribution in $E_{A}^{T}$ is less pronounced but still of the order of $20 \%$. Further studies with chains of different lengths indicate that the memory contribution in $E_{A}^{T}$ also depends on the chain length. In both flexible and stiff chains, this contribution decreases along with an increasing chain length and is almost negligible in the limit of very long chains. Finally, we close this work by discussing our results in light of recent experimental data ${ }^{28}$ as regards two-dimensional (2D) diffusion of very long DNA molecules on biological interfaces.

\section{MODEL POLYMER SYSTEM}

We consider a model of long chainlike molecules on smooth surfaces. The chains are modeled by the twodimensional fluctuating-bond (FB) model, ${ }^{24,25}$ in which the flat substrate is discretized such that each of the segments occupies four lattice sites, whose double occupancy is forbidden. The coverage is therefore defined as $\theta$ $\equiv 4 N N_{\mathrm{FB}} / L^{2}$, where $N$ is the number of chains and $L$ is the linear size of the system. $N_{\mathrm{FB}}$ is the number of segments in each chain (monodisperse case), which in this work is varied between 3 and $100 .{ }^{29}$ Studies at a finite coverage were carried out with $N_{\mathrm{FB}}=5$, while longer chains were examined in the case of a single diffusing chain (see Sec. IV C). Periodic boundary conditions are employed. The bond lengths $b_{l}$ (between two neighboring segments in a chain) are allowed to vary in the range $2 \leqslant b_{l} \leqslant \sqrt{13}$ in lattice units, where the upper limit prevents bonds from crossing each other. With these restrictions each segment can occupy 36 different lattice sites and there are 28 different bond angles $\phi \in[0, \pi)$, thus yielding a reasonable approximation for the continuum behavior. ${ }^{25}$ Furthermore, in the limit of only a single polymer, the tracer diffusion follows Rouse dynamics $\left(D_{T}\right.$ $\sim 1 / N_{\mathrm{FB}}$, describing polymer motion in the absence of hydrodynamic interactions and entanglements), as desired. ${ }^{30}$ Being also numerically very efficient, it is no wonder that the
FB model has been successfully applied to a number of problems in many-chain systems, ${ }^{25}$ including studies of dynamic quantities such as diffusion and viscosity. ${ }^{26,31-33}$

Concerning the interactions, the exclusion rules lead to an entropic repulsion between the polymers and to a coverage dependence of the collective diffusion coefficient, ${ }^{32,33}$ in agreement with experimental observations for some polymer systems. ${ }^{34,35}$ In this work, we have complemented the exclusion effects by adding direct intrachain interactions that govern the stiffness of a chain. To this end, we have used the following Hamiltonian:

$$
\frac{\mathcal{H}_{\mathrm{eff}}}{k_{B} T}=-\frac{J}{k_{B} T} \sum_{i=1}^{N} \sum_{j=1}^{N_{\mathrm{FB}}-2} \cos \phi_{i, j},
$$

where $J$ is the interaction strength, and the cosine is expressed in terms of the angle $\phi_{i, j}$ between neighboring bonds $j$ (from segment $j$ to $j+1$ ) and $j+1$ (from segment $j+1$ to $j+2)$ of chain $i$ such that $\phi_{i, j}=0$ if the two bonds are in parallel. Dynamics is introduced in the model by Metropolis moves of single segments, with a probability of acceptance $\min \left[e^{-\Delta \mathcal{H}_{e f f} / k_{B} T}, 1\right]$, where $\Delta \mathcal{H}_{\text {eff }}$ is the energy difference between final and initial configurations for acceptable moves, for which site exclusion and bond length restrictions must be satisfied. Use of Metropolis moves to simulate dynamics has been criticized in the past because this technique does not possess all the mathematical properties required to generate completely realistic dynamics. ${ }^{36}$ In this regard, the Kawasaki dynamics would be preferable ${ }^{36}$ although its acceptance rate is not as high as the one in Metropolis dynamics. Therefore, to make sure that the Metropolis dynamics chosen in this work is a justified approach to study qualitative aspects of the motion of chainlike particles, we have repeated some of the calculations for tracer diffusion using Kawasaki dynamics. We have found that this approach gives results qualitatively consistent with those obtained by Metropolis moves. ${ }^{37}$

The time scale in the simulations is defined as one Monte Carlo step (MCS), during which one attempts to move each segment of all chains once on the average. All transitions are between nearest neighbor sites. The simulations were started from a random initial configuration in which all exclusion rules and bond length restrictions are satisfied. The system was then equilibrated with large $J / k_{B} T$ by letting the system evolve for a long time, during which we followed the temporal decay of various quantities (such as the end-to-end distance of chains and the energy of the system) towards their equilibrium values. Typically about $10^{4} \mathrm{MC}$ steps were needed to get to equilibrium, although in practice considerably larger equilibration times were used (especially for large $N_{\mathrm{FB}}$ and large $\theta$ ). The configuration obtained in this fashion was then used as an initial configuration in a system with smaller $J / k_{B} T$, which in turn was equilibrated in a similar way.

In the present study we will concentrate on the coverage dependence of $D_{T}$ (defined in Sec. IV) with various degrees of stiffness, which is essentially determined by the ratio $J / k_{B} T$. For large values of $J / k_{B} T$, the chain acts like a rigid rod, while small values correspond to a flexible chain which is acting like a self-avoiding random walk. Further decrease of $J / k_{B} T$ to its negative region leads to a collapsed chain. In 
the simulations, we fix the temperature to a certain value (say, $k_{B} T=1$ ) and then vary the chain stiffness in terms of $J$. In studies of Arrhenius behavior with various degrees of chain stiffness, in turn, $J$ is held constant while $k_{B} T$ is slightly varied around its chosen value.

We now wish to comment on a few practical choices made in the present model approach. The key point is to simplify the system as much as possible, since otherwise studies of dynamic quantities such as the diffusion coefficient in a many-chain system with long chains would simply be out of reach. Therefore, the first choice we have made is to neglect direct interchain interactions. This assumption is in accord with experimental studies of $n$-alkanes, ${ }^{19-21}$ where one concluded that interchain interactions are relatively weak. The second simplification concerns the absence of interaction between the substrate and the adsorbate. This is not a major problem either, however, since for long chain molecules the mismatch between the molecule and the substrate becomes so pronounced that the substrate potential felt by the chain molecule becomes less and less corrugated. ${ }^{11,12,15,16}$ This is often the case in physisorption systems, and it has also been observed in cluster diffusion on a solid substrate. ${ }^{38}$ The adsorption of segments of long chains is therefore delocalized, which in the present model is reflected in the fact that the lattice sites occupied by the segments do not correspond to real adsorption sites but are rather an effective representation of the chain motion on a plane. ${ }^{39}$

We conclude that the present approach of using the FB model in characterizing the tracer diffusion process does not account for all microscopic degrees of freedom but is a coarse-grained approach, whose aim is to describe generic features of diffusion of many-chain systems in a relatively simple and efficient manner.

\section{STATIC PROPERTIES}

This section is concerned with static properties of chain structures that arise from the Hamiltonian in Eq. (1). Thus we aim to analyze the phase behavior, which then acts as a basis for understanding the dynamics discussed in Sec. IV. We expect that the absence of interchain interactions in the Hamiltonian leads to a liquidlike phase, which is characterized by positional disorder of the chains. However, just like in liquid crystals or Langmuir monolayers, the orientational order of single chains at high coverages may lead to orientational ordering even in a liquidlike system.

To characterize the order of individual chains, we consider the orientational order parameter,

$$
S \equiv \frac{1}{N_{\mathrm{FB}}-2} \sum_{j=1}^{N_{\mathrm{FB}}-2}\left\langle 2 \cos ^{2} \phi_{j}-S_{\mathrm{lim}}\right\rangle,
$$

where $\phi_{j}$ is an angle between bonds $j$ and $j+1$ in a chain, and \langle\rangle denotes a configuration average at a coverage studied. The quantity $S_{\text {lim }}$ is the athermal limit of $\left\langle 2 \cos ^{2} \phi_{j}\right\rangle$. In continuous systems with no self-avoidance between chain segments, $S_{\text {lim }}$ is simply one. In the present discrete case, however, one obtains a coverage dependent $S_{\text {lim }}$ which needs to be calculated separately. This guarantees that $S=0$ for ran-

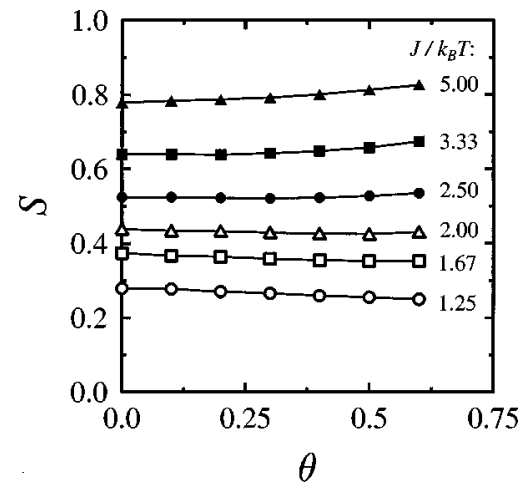

FIG. 1. Results for the orientational order parameter $S$ of single chains vs coverage $\theta$, when the chain stiffness is controlled in terms of the ratio $J / k_{B} T$ from the flexible regime $\left(J / k_{B} T=1.25\right)$ to the stiff one $\left(J / k_{B} T=5\right)$.

dom orientations in the limit $J / k_{B} T \rightarrow 0$. For perfectly linear chains we get $S=1$ after a proper normalization of Eq. (2).

Results shown in Fig. 1 confirm our expectation that the orientational order of single chains is mainly determined by the ratio $J / k_{B} T$. This is in agreement with a previous study, ${ }^{40}$ in which one used a harmonic bending potential to study semiflexible polymers. This approach [of replacing $\cos \phi_{i, j}$ in Eq. (1) with $\left.\phi_{i, j}^{2}\right]$ relates $J / k_{B} T$ to the persistence length of single chains, which indeed is a measure of orientational order of single chains. We find that, for stiff chains, an increase in the coverage leads to an increase in $S$. It is plausible that this is due to orientational ordering of different chains, which tend to pack themselves in a regular fashion, and is supported by configuration snapshots in Fig. 2. The behavior is different in the case of flexible chains, where $S$ tends to decrease slightly vs $\theta$. This seems to be a fingerprint of very flexible chains that tend towards a rounded shape, since $S_{\text {lim }}$ is also found to decrease with an increasing coverage. Nevertheless, in both limits we find that the tight packing suppresses thermal fluctuations in chain conformations and thus affects the orientational order parameter. Therefore, it is reasonable to expect that these packing requirements also influence interchain orientations. This idea is quantified by the orientational correlation function.

$$
C(r) \equiv\left\langle 2 \cos ^{2} \alpha-1\right\rangle,
$$

where $\alpha$ is an angle between the backbones of two distinct chains, whose centers of mass are a distance $r$ apart. $C(r)$ thus describes the decay of interchain orientational order vs distance. Results for $C(r)$ are consistent with previous conclusions, namely, $C(r)$ of flexible chains decays very rapidly and the orientational order between different chains dies out the more rapidly the larger is the coverage. In stiff chains, on the other hand, (short-range) orientational order is considerably more pronounced and it increases with an increasing coverage. To quantify these results in a simple manner, we show in Fig. 3 the correlation length $\xi$ as extracted from the asymptotic (intermediate) behavior $C(r) \sim \exp \left[-(r / \xi)^{2}\right] .^{41}$ This allows us to summarize the present section by concluding that there are two distinctly different regions. For stiff chains, orientational order becomes more pronounced with an increasing coverage, while flexible chains favor a decrease in order. Both regions are yet liquidlike. 


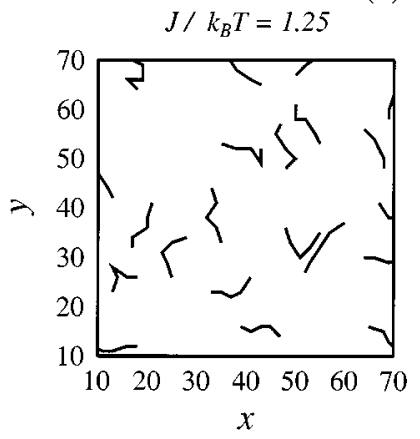

(a) $\theta=0.1$

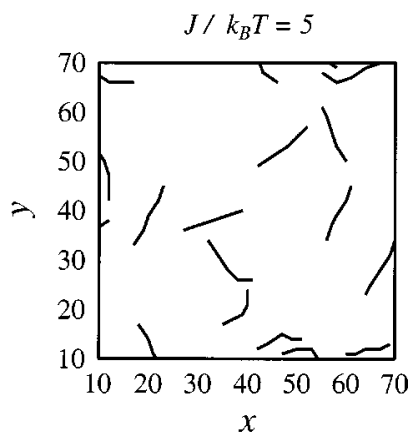

(b) $\theta=0.6$
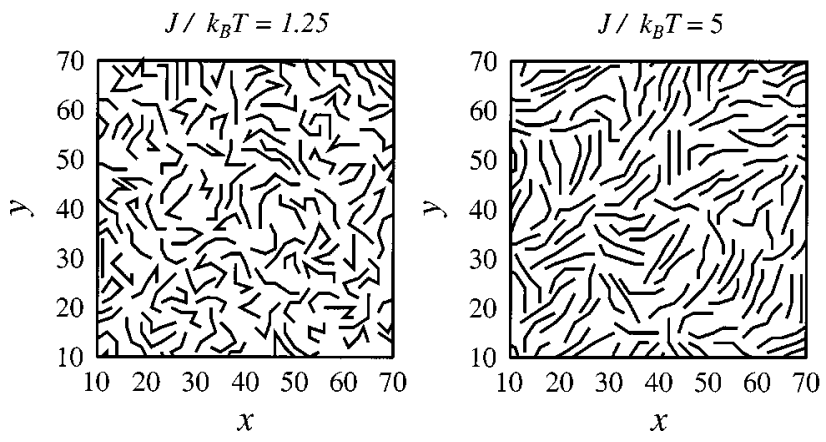

FIG. 2. Demonstrative configuration snapshots of the model polymer system at a coverage (a) $\theta=0.10$ and (b) $\theta=0.60$, with two degrees of flexibility $\left(J / k_{B} T=1.25\right.$ and $\left.J / k_{B} T=5\right)$ when the number of segments is chosen as $N_{\mathrm{FB}}=5$. Note that the figures are snapshots of a larger system and thus periodic boundary conditions are not seen correctly.

In the remaining part of this work, we study coverages $\theta \leqslant 0.6$ in which these conclusions are valid.

\section{TRACER DIFFUSION OF CHAINLIKE MOLECULES}

We consider the motion of a single tagged chain in the presence of other chains as a function of the coverage $\theta$. For fixed coverage in the hydrodynamic regime, it is characterized by the tracer diffusion coefficient ${ }^{1}$

$$
D_{T}=\lim _{t \rightarrow \infty} \frac{1}{4 N t} \sum_{i=1}^{N}\left\langle\left|\mathbf{r}_{i}(t)-\mathbf{r}_{i}(0)\right|^{2}\right\rangle
$$

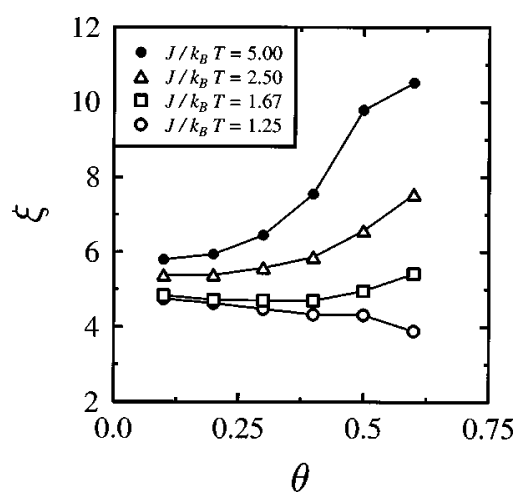

FIG. 3. Results for the correlation length $\xi$ as it comes from the interchain orientational correlation function $C(r)$ with $N_{\mathrm{FB}}=5$. The results for $\xi$ are given in lattice units. which accounts for all the diffusing chains $i=1, \ldots, N$, and is defined in terms of their center-of-mass positions $\mathbf{r}_{i}(t)$ at time $t$. We note that in general $D_{T}$ is a tensor quantity. Since diffusion in this system is isotropic, we use a simple scalar notation.

Within the lattice-gas description, which is the case in the FB model, a formally exact way of describing the temperature and coverage dependent tracer diffusion coefficient $D_{T}(\theta, T)$ is to write it as ${ }^{42-44}$

$$
D_{T}(\theta, T)=\frac{a^{2}}{4} \Gamma(\theta, T) f_{T}(\theta, T),
$$

where $a \equiv 1$ is the length of individual (nearest neighbor) displacements and $\Gamma(\theta, T)$ is the average transition rate of the center-of-mass of a chain. In other words, given a chain whose individual segments attempt to make displacements to neighboring sites, $\Gamma$ is their average success probability. ${ }^{39}$ The term $f_{T}(\theta, T)$ is a correlation factor containing all memory effects that arise from intrachain and interchain interactions, and essentially describes the extent of correlations between transitions, when the chain moves by a series of local chain displacements. In previous studies, substrate vibrations have also been shown to give rise to memory effects that affect the motion of a single adatom such that it can no longer be considered as a simple random walk. ${ }^{45,46}$ Since the substrate is static within the lattice-gas approach, these memory effects are not included in $f_{T}(\theta, T)$.

The correlation factor $f_{T}$ is very difficult to calculate analytically for an interacting system, and thus numerical simulations have usually been employed (see discussion and references in Ref. 27). The approximation where $f_{T} \equiv 1$ is called the dynamical mean field (DMF) theory for $D_{T} \cdot{ }^{33,44}$ This corresponds to a situation where all movements of chain segments are completely uncorrelated. In previous studies for athermal chains and in the presence of a direct attractive interaction between different chains, we have found ${ }^{33}$ that the DMF approximation yields a qualitatively correct description for the collective diffusion coefficient, ${ }^{1-3,43}$ while in tracer diffusion the DMF approach does not work as well. Here we use the decomposition in Eq. (5) to estimate the importance of actual memory effects via the correlation factor $f_{T}$ and the importance of memory effects in the effective diffusion barriers.

\section{A. Overall behavior of $D_{T}$ and memory effects}

The tracer diffusion coefficient $D_{T}$ and the rate $\Gamma$ were numerically computed throughout the coverage range with various ratios $J / k_{B} T$. The results for $D_{T}$ and $a^{2} \Gamma / 4$ are shown in Figs. 4(a) and 4(b). We first observe that $D_{T}$ decreases with increasing coverage. This behavior simply reflects the blocking effect of sites occupied by other chains and is in agreement with recent molecular dynamics simulations of $n$-butane $\mathrm{e}^{17,18}$ and $n$-hexane ${ }^{18}$ molecules, and with experiments on 2D diffusion of DNA oligonucleotides on coated glass plates. ${ }^{47,48}$ Another and more interesting observation is that $D_{T}$ and the DMF approximation $a^{2} \Gamma / 4$ are not in good agreement. This implies that the memory effects in the present case are of great importance. This is reflected in the correlation factor $f_{T}$ shown in Fig. 4(c). Unlike in the 


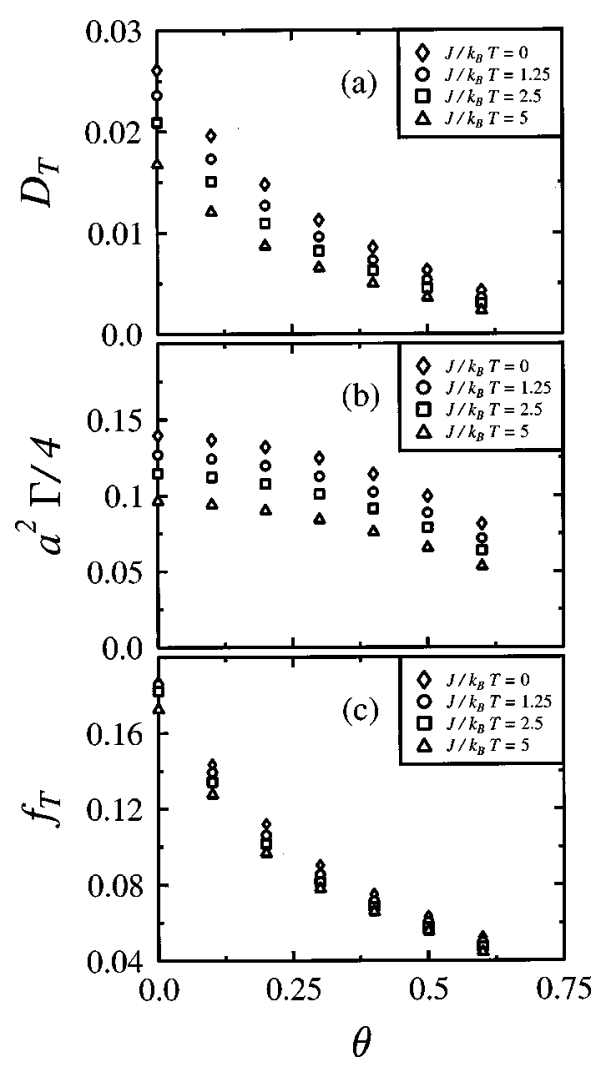

FIG. 4. (a) Results for the coverage dependence of the tracer diffusion coefficient $D_{T}$ with various degrees of chain stiffness $\left(J / k_{B} T\right.$ ranging from 1.25 to 5). (b) Corresponding results for the DMF approximation $a^{2} \Gamma / 4$, and (c) the resulting correlation factor $f_{T}$ [see Eq. (5)]. Results for the athermal case $\left(J / k_{B} T \rightarrow 0\right)$ are also given for comparison purposes.

case of adatom diffusion, where $f_{T}$ tends to one in the lowcoverage limit $\theta \rightarrow 0,{ }^{27}$ here $f_{T}$ is very small even in the limit of a single diffusing chain. This is an indication of memory effects as they arise from the correlated motion of individual segments in a single chain. The connectivity of chain segments therefore governs their motion considerably. As the coverage is increased, we furthermore find from Fig. 4(c) that the correlation factor $f_{T}$ decreases monotonously and thus the memory effects become more and more important.

The underlying mechanism that gives rise to the memory effect is the back-correlation principle observed also in previous studies for similar model systems. ${ }^{49,50}$ The backcorrelation mechanism originates from an idea that, after an initial displacement by some diffusing particle, a backward displacement of the particle is more likely than jumps to other sites. Based on various cases where this mechanism has been observed, ${ }^{17,27,49-51}$ it is plausible that it is a generic feature of dissipative 2D systems. Further studies regarding this idea and the related power-law decay of velocity correlations are currently in progress. ${ }^{50}$

\section{B. Memory effects in the effective diffusion barriers}

A convenient way to analyze the temperature dependence of the diffusion coefficient is to fit it to the activated Arrhenius form, which has been found to describe the surface diffusion data of short chainlike molecules reasonably well in various experimental ${ }^{19-21}$ and simulation ${ }^{7-9,11,12,16,17}$ studies. In this context, it is often assumed that the effective activation barrier $E_{A}^{T}$ of tracer diffusion arises entirely from the thermally activated nature of chain movements. However, one needs to keep in mind that the diffusion process arises from the motion of various individual chain segments, whose displacements are obviously correlated as is evident from the previous section. Therefore, the temperature dependence of the corresponding correlation factor $f_{T}(\theta, T)$ results in an additional contribution to $E_{A}^{T}$. This is indeed the case in our model system as shown in Fig. 4(c), where $f_{T}$ demonstrates a significant dependence on the chain flexibility via $J / k_{B} T$ (note the differences between adjacent curves).

To characterize the importance of memory effects in the observed activation barriers, we follow the common practice in defining the effective tracer diffusion barrier $E_{A}^{T}$ as the local slope of $D_{T}$ in an Arrhenius plot,

$$
E_{A}^{T}(\theta) \equiv-\frac{\partial}{\partial\left(1 / k_{B} T\right)} \ln D_{T}(\theta, T) .
$$

In a similar fashion, we define a displacement rate barrier $E_{A}^{\Gamma}(\theta)$ as the local slope of $\ln \Gamma(\theta)$ vs $1 / k_{B} T$. Within the lattice-gas description, the effective tracer diffusion barrier therefore comes from two distinct sources,

$$
E_{A}^{T}=E_{A}^{\Gamma}+E_{A}^{f_{T}},
$$

where it needs to be emphasized that $E_{A}^{f_{T}}$ should not be interpreted as an actual "activation barrier," since $f_{T}$ is not a thermally activated quantity. Rather, $E_{A}^{f_{T}}$ just describes the temperature dependence of the correlation factor.

The effective barriers $E_{A}^{T}$ and $E_{A}^{\Gamma}$ are shown in Figs. 5(a) and 5(b) with various degrees of flexibility. The qualitative similarity between $E_{A}^{T}$ and $E_{A}^{\Gamma}$ is evident, but so is also their quantitative disagreement. This difference is entirely due to the memory effects.

As shown in Fig. 5(c), the role of memory effects in $E_{A}^{T}$ is most important in flexible chains at finite coverages. Then we find that up to about $40 \%$ of the effective barrier $E_{A}^{T}$ comes from the memory contribution, which arises from temperature variations in $f_{T}$ [see Fig. 4(c)]. Even in the limit of just a single diffusing chain, the memory contribution to the effective barrier $E_{A}^{T}$ is about $15 \%$, which highlights the importance of intrachain correlations in the motion of a chain. In the case of stiff chains, the memory effects are playing a less significant role. Then the contribution due to memory effects in $E_{A}^{T}$ is approximately about $20 \%$, although its coverage dependence is rather strong.

The importance of memory effects in the tracer diffusion barrier raises an interesting question of how well this memory contribution can be traced back to the coverage dependence of the correlation factor $f_{T}$. This idea is supported by recent studies in a system of single adatoms diffusing on a solid substrate, where the results indeed suggest that the contribution of memory effects in $E_{A}^{T}$ directly correlates with the coverage dependence of $f_{T} \cdot{ }^{23,27}$ However, there is reason to stress that the memory contribution in $E_{A}^{T}$ describes the temperature dependence of memory effects, and therefore the connection is far from trivial. It turns out that in flexible chains the memory contribution in $E_{A}^{T}$ increases with an in- 


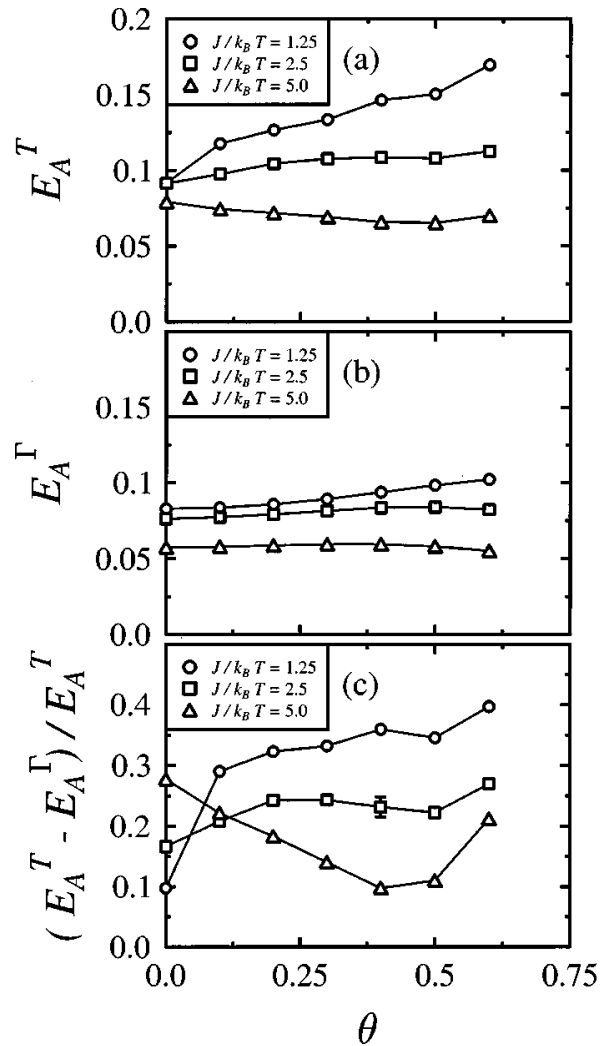

FIG. 5. The effective barriers of (a) $E_{A}^{T}$ and (b) $E_{A}^{\Gamma}$ vs the coverage $\theta$ with a few degrees of flexibility $J / k_{B} T$. (c) Corresponding results for the relative importance of memory effects in the tracer diffusion barrier as characterized by $\left[E_{A}^{T}-E_{A}^{\Gamma}\right] / E_{A}^{T}$.

creasing coverage [see Fig. 5(c)], in agreement with results in Fig. 4(c), where the behavior of $f_{T}$ implies that the memory effects become more pronounced at an increasing coverage. For stiff chains, the same idea works well in the single-chain limit $(\theta \rightarrow 0)$, where the memory effects in stiff chains are stronger than those in flexible ones [see Fig. 4(c)]. However, as the coverage increases, it turns out that this picture does not suffice to explain the situation completely. Namely, as Fig. 5(c) reveals, there is a crossover around $\theta \approx 0.10$ above which the temperature dependence of memory effects is most prominent in flexible chains. This demonstrates that, in general, the coverage and the temperature dependence of memory effects are not directly coupled but rather depend on the thermodynamic conditions in question.

Results above demonstrate that the effective tracer diffusion barrier $E_{A}^{T}$ is indeed a very complex quantity and, within the present model description, arises from two distinct sources. In addition to the contribution $E_{A}^{\Gamma}$ due to thermally activated displacement processes, there is also another important contribution arising from temperature dependent memory effects. This makes the interpretation of $E_{A}^{T}$ very difficult even in simple adsorption systems, and obviously even more complicated in systems where complex particles such as chainlike molecules are diffusing on a plane.

To understand the behavior of the effective diffusion barriers in a bit more detail, we briefly discuss the interpretation of $E_{A}^{\Gamma}$, which in the present case originates from intrachain interactions only. ${ }^{52}$ To start with, we make the usual

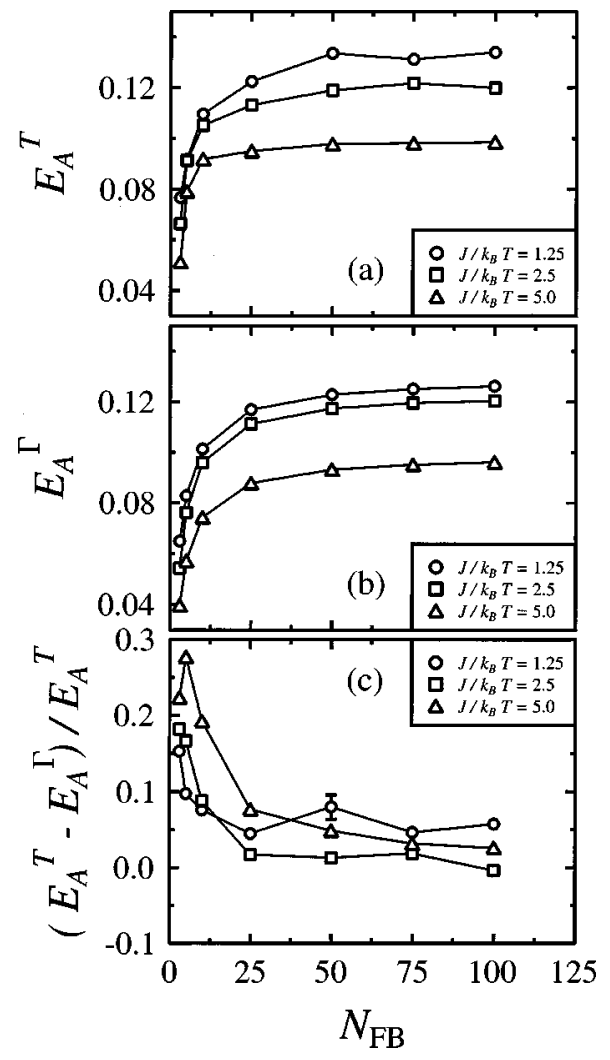

FIG. 6. The effective barriers of (a) $E_{A}^{T}$ and (b) $E_{A}^{\Gamma}$ vs chain length $N_{\mathrm{FB}}$ with a few degrees of flexibility $J / k_{B} T$. Here the results are for a single diffusing chain only. (c) Corresponding results for the relative importance of memory effects in the tracer diffusion barrier as characterized by $\left[E_{A}^{T}-E_{A}^{\Gamma}\right] / E_{A}^{T}$.

assumption that there is some activation process whose barrier is mostly dominating $E_{A}^{\Gamma}, 23,27,53$ which hence provides some qualitative information of predominant interactions. Now, we first note from Fig. 5(b) that the effective rate barrier of flexible chains is larger than the corresponding barrier of stiff chains. Based on the Hamiltonian in Eq. (1), this suggests that the motion of stiff chains does not involve large conformational changes but the chains prefer to maintain their rodlike shape and instantaneous rate barriers are thus relatively small (see the snapshots in Fig. 2). The motion of flexible chains with a curved shape, on the other hand, does involve large conformational changes whose instantaneous barriers are relatively large. The behavior of rate barriers in the present model system is therefore related to the orientational ordering of single chains, and is supported by the results in Fig. 5(b). For example, $E_{A}^{\Gamma}$ of very flexible chains increases monotonously as a function of coverage, which implies that the chains become more bent with an increasing coverage, in agreement with conclusions made in Sec. III. However, we wish to emphasize that although chain orientational order and rate barriers seem to be related, it is not clear whether this idea works in systems where direct interchain interactions are of importance.

\section{Effect of varying the chain length}

We close this section by briefly discussing the effect of chain length on the effective diffusion barriers. In Figs. 6(a) and 6(b), we show the barriers $E_{A}^{T}$ and $E_{A}^{\Gamma}$ for a single dif- 
fusing chain (in the zero-coverage limit $\theta \rightarrow 0$ ) vs the chain length $N_{\mathrm{FB}}$. It turns out that the behavior of flexible and stiff chains is qualitatively very similar. In both cases, the barriers increase monotonously with an increasing chain length and converge to some limiting value, which depends on $J / k_{B} T$. Increasing the chain length therefore has the same effect as increasing the chain flexibility. This result is consistent with previous molecular dynamics simulations ${ }^{7,8,12,15,16}$ and experimental work ${ }^{19}$ for short $n$-alkanes (up to $n$-octane), although a deviation from a monotonously increasing trend of the effective tracer diffusion barrier has also been observed. ${ }^{15,16}$ One suggested explanation for this deviation is a change in the diffusion mechanism, when the chain length exceeds some critical value. ${ }^{15}$ If this is the case, then the interactions between the substrate and the chain are clearly important. This aspect is not included in the present model system and could therefore explain the slight difference.

As far as the memory contribution in $E_{A}^{T}$ is concerned, results in Fig. 6(c) show that it is most prominent in stiff chains. Even in this case, however, the memory contribution decreases rather rapidly as the number of chain segments is increased, and converges to a value of a few percent at very large chain lengths. This result can be related to previous observations for collective diffusion of similar chains, ${ }^{33,44}$ where one studied the center-of-mass diffusion of the whole system and found that the corresponding memory effects are weak. In a similar fashion, the motion of distinct segments in a long chain is only weakly coupled, and thus the dynamical correlations in the center-of-mass motion of a single chain cancel to a large degree. This trend is most evident in flexible chains (with small $J / k_{B} T$ ) as the results in Fig. 6(c) also demonstrate. It is also intriguing to compare these results to recent experiments on 2D diffusion of DNA molecules. ${ }^{28} \mathrm{In}$ this study, Maier and Rädler found that conformations of DNA molecules bound to a fluidlike lipid bilayer supported on a glass substrate can be characterized by a $2 \mathrm{D}$ selfavoiding random walk, and that the tracer diffusion of these molecules follows Rouse dynamics $\left(D_{T} \sim 1 / N_{\mathrm{FB}}\right)$. These observations are fully consistent with the approach used in this work for small $J / k_{B} T$. Therefore, we can use our results to conclude that, for a single very long DNA molecule diffusing on a plane, the memory effects are not playing a significant role in the tracer diffusion barrier. The importance of memory effects in the diffusion barrier of similar chains at a large coverage remains to be studied. ${ }^{54}$

\section{SUMMARY AND DISCUSSION}

To summarize, we have studied the coverage dependence of surface diffusion for chainlike molecules by a model, which includes short-ranged excluded volume interactions between different chains as well as an intrachain bond angle potential to describe the chain stiffness. We have focused on the role played by chain stiffness and the resulting memory effects in tracer diffusion, and in particular studied their importance in the effective tracer diffusion barrier $E_{A}^{T}$ extracted from the Arrhenius form. We have found that the memory effects in tracer diffusion become more pronounced at an increasing coverage for both stiff and flexible chains. Increasing the chain flexibility has been found to have the same overall effect as increasing the chain length, namely, they both increase $E_{A}^{T}$. More detailed studies of $E_{A}^{T}$ have revealed that only a certain part of the tracer diffusion barrier arises from thermally activated chain segment displacements. Another important contribution comes from the temperature dependence of memory effects, which in the zero-coverage limit is found to be about $20 \%$ of $E_{A}^{T}$. This memory contribution is coverage dependent and can be as large as about $20 \%-40 \%$ at large coverages. However, we note that the memory contribution in the tracer diffusion barrier also depends on the chain length and tends to decrease with an increasing chain length. For very long chains, such as in 2D diffusion of long DNA molecules, our results therefore give support to an idea that the memory contribution is very small and the effective tracer diffusion barrier is associated with thermally activated chain motions.

We wish to note that, in the present model approach, the substrate has been assumed to be static. The memory effects we have studied have therefore arised from intrachain and interchain interactions only. In general, however, there is also another source of memory effects that arises from chain-substrate interactions. Namely, if the substrate particles move on a time scale that is comparable to the time scale of diffusive chains, then the two time scales cannot be separated and the substrate-chain interactions can give rise to additional memory effects that influence the motion of the chains. This is the case, for example, in various biologically interesting problems, where complex molecules diffuse along a flexible, fluctuating surface such as the membranesolvent interface. Such studies, where the two kinds of memory effects are competing and their relative importance is to be examined, would be most interesting but are beyond the scope of the present work.

\section{ACKNOWLEDGMENTS}

One of the authors (T. H.) thanks Helsinki Institute of Physics at the University of Helsinki for computing resources. Juha Merikoski is thanked for useful discussions. This work has, in part, been supported by a grant from the European Union (I. V.).

\footnotetext{
${ }^{1}$ R. Gomer, Rep. Prog. Phys. 53, 917 (1990).

${ }^{2}$ Surface Diffusion: Atomistic and Collective Processes, edited by M. C. Tringides (Plenum, New York, 1997).

${ }^{3}$ T. Ala-Nissila and S. C. Ying, Prog. Surf. Sci. 39, 227 (1992).

${ }^{4}$ G. L. Kellogg, Surf. Sci. Rep. 21, 1 (1994), and references therein.

${ }^{5}$ T. R. Linderoth, S. Horch, L. Petersen, S. Helveg, E. Lægsgaard, I. Stensgaard, and F. Besenbacher, Phys. Rev. Lett. 82, 1494 (1999); F. Montalenti and R. Ferrando, ibid. 82, 1498 (1999), and references therein.

${ }^{6}$ J. Merikoski, I. Vattulainen, J. Heinonen, and T. Ala-Nissila, Surf. Sci. 387, 167 (1997), and references therein.

${ }^{7}$ D. Cohen and Y. Zeiri, J. Chem. Phys. 97, 1531 (1992).

${ }^{8}$ D. Cohen and Y. Zeiri, Surf. Sci. 274, 173 (1992).

${ }^{9}$ Y. Zeiri, Can. J. Chem. 72, 813 (1994).

${ }^{10}$ M. Silverberg, J. Chem. Phys. 99, 9255 (1993).

${ }^{11}$ K. A. Fichthorn, P. G. Balan, and Y. Chen, Surf. Sci. 317, 37 (1994).

${ }^{12}$ D. Huang, Y. Chen, and K. A. Fichthorn, J. Chem. Phys. 101, 11021 (1994).

${ }^{13}$ J. S. Raut and K. A. Fichthorn, J. Chem. Phys. 103, 8694 (1995).

${ }^{14}$ J.-C. Wang and K. A. Fichthorn, Langmuir 12, 139 (1996).

${ }^{15}$ K. A. Fichthorn, Adsorption 2, 77 (1996).

${ }^{16}$ J. S. Raut and K. A. Fichthorn, J. Chem. Phys. 108, 1626 (1998).
} 
${ }^{17}$ J. S. Raut, D. S. Sholl, and K. A. Fichthorn, Surf. Sci. 389, 88 (1997).

${ }^{18}$ J. S. Raut and K. A. Fichthorn, J. Chem. Phys. 110, 587 (1999).

${ }^{19}$ J. L. Brand, M. V. Arena, A. A. Deckert, and S. M. George, J. Chem. Phys. 92, 5136 (1990).

${ }^{20}$ M. V. Arena, A. A. Deckert, J. L. Brand, and S. M. George, J. Phys. Chem. 94, 6792 (1990).

${ }^{21}$ M. V. Arena, E. D. Westre, and S. M. George, J. Chem. Phys. 94, 4001 (1991).

${ }^{22}$ L. J. Lauhon and W. Ho, J. Chem. Phys. 111, 5633 (1999).

${ }^{23}$ I. Vattulainen, Surf. Sci. 412-413, L911 (1998).

${ }^{24}$ I. Carmesin and K. Kremer, Macromolecules 21, 2819 (1988).

${ }^{25}$ Monte Carlo and Molecular Dynamics Simulations in Polymer Science, edited by K. Binder (Oxford, New York, 1995).

${ }^{26}$ J. Baschnagel, W. Paul, V. Tries, and K. Binder, Macromolecules 31, 3856 (1998).

${ }^{27}$ I. Vattulainen, S. C. Ying, T. Ala-Nissila, and J. Merikoski, Phys. Rev. B 59, 7697 (1999).

${ }^{28}$ B. Maier and J. O. Rädler, Phys. Rev. Lett. 82, 1911 (1999)

${ }^{29}$ Typically one segment in the FB model corresponds to about five structural units in a real linear molecule (Refs. 25,26 ), so roughly speaking our study (with chains of length 3-100 segments) corresponds to a degree of polymerization of about $15-500$.

${ }^{30}$ M. Doi, Introduction to Polymer Physics (Oxford, New York, 1996).

${ }^{31}$ V. Tries, W. Paul, J. Baschnagel, and K. Binder, J. Chem. Phys. 106, 738 (1997).

${ }^{32}$ T. Ala-Nissila, S. Herminghaus, T. Hjelt, and P. Leiderer, Phys. Rev. Lett. 76, 4003 (1996).

${ }^{33}$ T. Hjelt, S. Herminghaus, T. Ala-Nissila, and S. C. Ying, Phys. Rev. E 57, 1864 (1998).

${ }^{34}$ C. H. Mak, B. G. Koehler, and S. M. George, J. Vac. Sci. Technol. A 6, 856 (1988); U. Albrecht, A. Otto, and P. Leiderer, Phys. Rev. Lett. 68, $3192(1992)$

${ }^{35}$ S. Herminghaus, U. Sigel, U. Albrecht, and P. Leiderer, in Proceedings of the XVth Moriond Workshop "Short and Long Chains at Interfaces," Condensed Matter Physics Series, edited by J. Daillont et al. (Villars-surOllon, Switzerland, 1995)

${ }^{36}$ K. A. Fichthorn and W. H. Weinberg, J. Chem. Phys. 95, 1090 (1991).

${ }^{37}$ T. Hjelt and I. Vattulainen (unpublished, 1999); To further justify our approach, we wish to point out that the present model approach has been used in studies of collective diffusion of chainlike particles, in which one has found (Ref. 32) results qualitatively consistent with experimental observations (Ref. 35). Furthermore, related FB model studies of droplet spreading (with Metropolis moves) have given results (Refs. 32, 33) in agreement with experiments [U. Albrecht, A. Otto, and P. Leiderer, Phys. Rev. Lett. 68, 3192 (1992); Surf. Sci. 283, 383 (1993)] and recent mo- lecular dynamics simulations [J. M. Lahtinen, T. Hjelt, and T. Ala-Nissila, Surf. Sci. (in press).

${ }^{38}$ P. Deltour, J.-L. Barrat, and P. Jensen, Phys. Rev. Lett. 78, 4597 (1997).

${ }^{39}$ We wish to emphasize that our main objective is to estimate the importance of memory effects. This requires that the chain motion is described in terms of well-defined, distinct displacements. In a real system, these displacements could correspond to gauche-trans transitions, for example, between which the monomer in question resides in the corresponding potential well for a time long compared to the transition. In the present coarse-grained system, the situation is more complicated but the same idea still holds, and thus the lattice-gas approach is justified at least on an operational basis.

${ }^{40}$ M. Dijkstra and D. Frenkel, Phys. Rev. E 50, 349 (1994).

${ }^{41}$ In the isotropic phase, one would expect to find an exponential decay of $C(r)$. In the present work, however, the decay was better characterized by a Gaussian at intermediate ranges $r$. Use of an exponential decay to fit $C(r)$ results in correlation lengths that are qualitatively consistent with those in Fig. 3, however.

${ }^{42}$ D. A. Reed and G. Ehrlich, Surf. Sci. 102, 588 (1981)

${ }^{43}$ A. Danani, R. Ferrando, E. Scalas, and M. Torri, Int. J. Mod. Phys. B 11, 2217 (1997)

${ }^{44}$ T. Hjelt, I. Vattulainen, J. Merikoski, T. Ala-Nissila, and S. C. Ying, Surf. Sci. 380, L501 (1997); 402-404, 253 (1998).

${ }^{45}$ A. F. Voter and J. D. Doll, J. Chem. Phys. 82, 80 (1985).

${ }^{46}$ A. Cucchetti and S. C. Ying, Phys. Rev. B 54, 3300 (1996).

${ }^{47}$ V. Chan, D. J. Graves, P. Fortina, and S. E. McKenzie, Langmuir 13, 320 (1997).

${ }^{48}$ V. Chan, S. E. McKenzie, S. Surrey, P. Fortina, and D. J. Graves, J. Colloid Interface Sci. 203, 197 (1998).

${ }^{49}$ S. C. Ying, I. Vattulainen, J. Merikoski, T. Hjelt, and T. Ala-Nissila, Phys. Rev. B 58, 2170 (1998)

${ }^{50}$ T. Hjelt, I. Vattulainen, T. Ala-Nissila, and S. C. Ying, Surf. Sci. Lett. (in press).

${ }^{51}$ P. B. S. Kumar and M. Rao, Phys. Rev. Lett. 77, 1067 (1996); see also discussion by C. P. Lowe, R. Van Roij, and D. Frenkel, ibid. 79, 1168 (1997); P. B. S. Kumar and M. Rao, ibid. 79, 1169 (1997).

${ }^{52}$ Close to (continuous) phase transition boundaries, the rate barrier $E_{A}^{T}$ can also include contributions from entropy changes in a system (Refs. 23, 27). In this work, such effects are not playing a role.

${ }^{53}$ J. W. Haus and K. W. Kehr, Phys. Rep. 150, 263 (1987).

${ }^{54}$ Due to a huge numerical effort required for studies of very long chains at a large coverage within the Arrhenius description, this case was not studied in this work. 